\title{
Suboptimal Vitamin D levels among adult survivors of childhood cancers
}

\author{
Denise A. Rokitka' ${ }^{1}$, Robert Lisac ${ }^{1}$, Jennifer E Heffler ${ }^{1}$, Michael A Zevon ${ }^{2}$, Martin A Mahoney ${ }^{2,3}$ \\ ${ }_{1}^{1}$ Department of Pediatric Oncology, Roswell Park Cancer Institute, Buffalo, New York, USA \\ ${ }^{2}$ Department of Medicine, Roswell Park Cancer Institute, Buffalo, New York, USA \\ ${ }^{3}$ Department of Family Medicine, State University at Buffalo, New York, USA
}

Received March 10, 2016; Revised July 13, 2016; Accepted July 14, 2016; Published Online July 16, 2016

\section{Original Article}

\begin{abstract}
Purpose: Vitamin D plays an important role in many bodily systems, with increasing evidence suggesting its importance for the prevention of chronic diseases and cancer. The identification of vitamin D levels in childhood cancer survivors becomes, therefore, particularly relevant, given that optimizing levels may contribute to the prevention of secondary malignancies and chronic diseases. Methods: A cross - sectional analysis of serum 25 - hydroxyvitamin D levels among adult survivors of childhood cancers living in New York State and surrounding areas $(\mathrm{n}=139)$ was performed. Independent variables included gender, race/ethnicity, cancer site, year of diagnosis, past medical and surgical history, prior radiation therapy; prior chemotherapy, age at diagnosis, age at last clinic visit, year of last clinic visit, height, weight, body mass index, and vitamin D supplementation. Results: Overall, 34\% of survivors were vitamin D deficient $(<$ $20 \mathrm{ng} / \mathrm{ml}), 39 \%$ were classified as insufficient $(20-29 \mathrm{ng} / \mathrm{ml})$ and $27 \%(\geq 30$ $\mathrm{ng} / \mathrm{ml}$ ) were classified as having sufficient levels. Despite vitamin D supplementation among 41 patients, $68.3 \%$ continued to have insufficient or deficient levels. Participants with a BMI $>25$ demonstrated lower levels of vitamin D ( $\mathrm{p}<0.05)$. Vitamin D levels did not vary by age group, race, ethnicity, diagnosis, or years since diagnosis. Conclusion: Given the growing awareness of the role of vitamin $\mathrm{D}$ and the documented late effects of treatment for childhood cancers, the high prevalence of vitamin D deficiency within the childhood cancer survivor population is of concern. Vitamin D represents an important target for surveillance and intervention to help improve long - term outcomes of childhood cancer survivors.
\end{abstract}

Keywords: Vitamin D deficiency, Cancer survivor, Pediatrics, Preventive care, Quality of care

\section{Introduction}

The success in treating childhood and adolescent cancers is evident by the dramatic reduction in mortality rates over the past several decades, with more than $80 \%$ of current patients now surviving for five years. ${ }^{1,2}$ This achievement, however, has led to a growing number of long - term survivors with new complications from their previous therapies. An extended follow up by Mertens et $a l^{2}$, for example, found excess overall mortality of 8.4 fold when compared to age -, sex - and year - matched
US population controls. Excess mortality was due primarily to secondary malignancies, followed by cardiac and pulmonary causes. ${ }^{2}$ As a result, late effects of therapy and quality of life among adult survivors of childhood cancers have become areas of intense investigation. ${ }^{3,4}$

Given the critical need for structured follow-up of survivors of childhood cancer, evaluations should 
include assessment of important health-related factors such as levels of Vitamin D, a fat soluble vitamin which is involved in many physiologic pathways. Emerging evidence suggests that Vitamin D levels should be greater than $30 \mathrm{ng} / \mathrm{ml}$ to achieve the desired health effects. ${ }^{5}$ A data brief issued by the National Center for Health Statistics (NCHS) provides Vitamin D levels as assessed in the National Health and Nutrition Examination Surveys (NHANES). Findings from this survey indicate that approximately one-third of the general population were at risk of Vitamin D deficiency with levels $<20 \mathrm{ng} / \mathrm{ml} .{ }^{6}$ In a study by Forrest et al, Vitamin D status was examined using the NHANES data. Overall prevalence of Vitamin D deficiency $(\leq 20 \mathrm{ng} / \mathrm{mL})$ was found to be 41.6\%; with African Americans presenting with the highest rate of Vitamin D deficiency at $82.1 \%$ and Caucasians at $30.9 \%$. Furthermore, a significant link was found between those participants that were Vitamin D deficient and had less than a college education, poor health status, hypertension, low high-density lipoprotein cholesterol levels, and those not consuming milk. ${ }^{7}$ A comprehensive review conducted by Holick along with other research has found a significant increase in long term cancer risk among individuals with deficient vitamin D levels. ${ }^{8-12}$ As is clear from these findings, deficiency states are of great concern given the evidence suggesting that adequate Vitamin D levels may be important for prevention and management of type 1 and type 2 Diabetes mellitus, hypertension, and cancer. ${ }^{13-16}$ However, research on the clinical impact of screening and treatment of Vitamin D remains inadequate. Several studies in Acute Lymphocytic Leukemia survivors have shown that patients with exposure to corticosteroids have increased bone demineralization and poor mineral homeostasis. ${ }^{17-19}$ This suggests that childhood cancer survivors may be at an increased risk of Vitamin D deficiency.

Given the growing awareness of the role of Vitamin D and the documented late effects associated with treatment of childhood cancer, the issue of Vitamin D levels in the childhood cancer survivor population is of great relevance. Childhood cancer survivors are an ideal group to study Vitamin D levels, since optimizing levels may help to contribute to the prevention of secondary malignancies and chronic diseases. Childhood cancer survivors experience an increased risk of developing secondary malignancies and other chronic illnesses as a result of prior therapies. This study examines the prevalence of Vitamin D deficiency among adult survivors of childhood cancers.

\section{Methods and Materials}

Roswell Park Cancer Institute operates a Long-Term Follow - Up (LTFU) Center as part of the Department of Pediatrics which provides specialized surveillance, medical care, and counseling to former childhood cancer patients. Patients who are more than 5 years from their cancer diagnosis are seen in this outpatient center.

A cross - sectional medical chart review was utilized to abstract selected variables from patients seen in the LTFU center between 2009 and 2014. Two hundred and three patients were seen at 383 separate office visits over the period of 5 years. Sixty-four patients (31.5\%) were excluded due to a lack of data on vitamin D levels leaving 139 patients (68.5\%) with at least one measurement of Vitamin D who were included in the analytic data set. Among these 139 patients, 70 patients (50.4\%) had Vitamin D levels available for at least 2 visits.

The main dependent variable was serum 25 hydroxyvitamin D. The first Vitamin D level during the observation period was used for the analysis. Vitamin D sufficiency was defined as a serum 25 - hydroxy vitamin D level $\geq 30 \mathrm{ng} / \mathrm{ml}$, insufficiency as a level from $20 \mathrm{ng} \mathrm{ml}$ to $29.9 \mathrm{ng} / \mathrm{ml}$, and deficiency as $\leq 20 \mathrm{ng} / \mathrm{ml}$.

Independent variables included gender, race/ethnicity (non-Hispanic white versus all other), cancer site (leukemia, lymphoma, sarcoma, Central Nervous System (CNS), other), year of cancer diagnosis, past medical history, past surgical history, prior radiation therapy; prior chemotherapy, zip code of residence, age at diagnosis, age at last clinic visit, calendar year of last clinic visit, height, weight, body mass index (BMI, normal BMI $<25$, overweight BMI $25-29.9$, and obese BMI $\geq$ 30), and self - reported Vitamin D supplementation / use of multivitamins. Seasons were categorized as Winter (December 1 - February 28), Spring (March 1 - May 31), Summer (June 1 - August 31), and Fall (September 1 November 30 ).

SPSS Version 21 (@ IBM, Cary, NC) was used to facilitate data analyses, which included descriptive summaries and a comparison of Vitamin D status by demographic characteristics and supplementation status using chi-square tests. Vitamin D levels by supplementation status were analyzed using the Mann Whitney - U test. Analysis of variance was used to examine the relationship between mean vitamin D levels and BMI. In addition, a logistic model was constructed to examine factors associated with Vitamin D deficiency. The protocol for this research project was approved by the Roswell Park Cancer Institute Institutional Review Board.

\section{Results}

As reported above, one hundred thirty-nine patients with at least one Vitamin D assessment were identified for analysis. Clinical characteristics of childhood cancer survivors are presented in Table 1. The mean serum 25 hydroxyvitamin D level for all patients was $26.6 \mathrm{ng} / \mathrm{ml}$ (median 25.2, range: 5.0 - 96.6). Overall, $34 \%$ of 
survivors were Vitamin D deficient, 39\% were classified as insufficient and $27 \%$ were classified as having sufficient Vitamin D levels. Chi - square testing revealed no significant difference in categorical Vitamin D status by gender, age group, race, cancer diagnosis, and years since diagnosis.

\subsection{The distribution of Vitamin D levels based on supplementation status is shown in Figure}

Median vitamin D levels among those supplemented (26.8 ng/ml) was greater than those not reporting supplementation $(21.2 \mathrm{ng} / \mathrm{ml}, \mathrm{p}=0.0016$, Mann Whitney $U$ test); however these median levels remain within the "insufficiency" range. Among the 41 patients who reported taking supplemental Vitamin D, 68.3\% continued with insufficient or deficient Vitamin D levels at the subsequent draw despite supplementation.

Table 1: Selected demographics and clinical characteristics of study subjects by gender, $\mathrm{n}=139$

\begin{tabular}{|c|c|c|c|c|c|}
\hline & $\begin{array}{c}\text { Male } \\
\mathrm{n}\end{array}$ & $(\%)$ & $\begin{array}{c}\text { Female } \\
n\end{array}$ & $(\%)$ & p-value* \\
\hline Age (in years) & & & & & 0.73 \\
\hline $18-39$ & 51 & $(76.1)$ & 53 & (73.6) & \\
\hline \multirow[t]{2}{*}{$40+$} & 16 & (23.9) & 19 & $(26.4)$ & \\
\hline & & & & & 0.2 \\
\hline \multicolumn{6}{|l|}{ Race/Ethnicity } \\
\hline Non-Hispanic White & 60 & $(89.6)$ & 59 & $(81.9)$ & \\
\hline Other & 7 & $(10.4)$ & 13 & (18.1) & \\
\hline & & & & & 0.39 \\
\hline \multicolumn{6}{|l|}{ Cancer Type } \\
\hline Leukemia & 28 & $(41.8)$ & 24 & $(33.3)$ & \\
\hline Lymphoma & 20 & (29.9) & 18 & $(25.0)$ & \\
\hline Sarcoma & 5 & $(7.5)$ & 13 & (18.1) & \\
\hline CNS & 7 & $(10.4)$ & 9 & $(12.5)$ & \\
\hline Other & 7 & $(10.4)$ & 8 & (11.1) & \\
\hline Years Since Diagnosis & & & & & 0.91 \\
\hline $0-9$ & 6 & $(9.0)$ & 8 & (11.1) & \\
\hline $10-19$ & 18 & $(26.9)$ & 21 & $(29.2)$ & \\
\hline $20-29$ & 24 & $(35.8)$ & 22 & $(30.6)$ & \\
\hline $30+$ & 19 & $(28.4)$ & 21 & $(29.2)$ & \\
\hline Body Mass Index & & & & & 0.89 \\
\hline$<25$ (normal) & 24 & $(35.8)$ & 27 & $(37.5)$ & \\
\hline $25-29.9$ (overweight) & 24 & $(35.8)$ & 23 & (31.9) & \\
\hline$\geq 30$ (obese) & 19 & $(28.4)$ & 22 & $(30.6)$ & \\
\hline \multicolumn{6}{|l|}{ Vitamin D Level (ng/mL) } \\
\hline Median & 22.2 & & 26.2 & & 0.28 \\
\hline Range & $5.0-96.6$ & & $7.8-77.6$ & & \\
\hline -deficient & 24 & $(36)$ & 23 & $(32)$ & \\
\hline -insufficient & 27 & $(40)$ & 27 & (38) & \\
\hline -adequate & 16 & $(24)$ & 22 & (31) & \\
\hline
\end{tabular}

* p-values calculated using Chi-Square test.

Table 2: Vitamin D status by gender and supplementation status.

\begin{tabular}{|c|c|c|c|c|c|c|c|}
\hline \multirow{3}{*}{ Deficient } & \multicolumn{3}{|c|}{ Male } & \multicolumn{4}{|c|}{ Female } \\
\hline & \multicolumn{2}{|c|}{ Supplement } & No Supplement & \multicolumn{2}{|c|}{ Supplement } & \multicolumn{2}{|c|}{ No supplement } \\
\hline & $6.7 \%$ & $(n=1)$ & $45.1 \% \quad(n=23)$ & $11.5 \%$ & $(n=3)$ & $45.5 \%$ & $(n=20)$ \\
\hline Insufficient & $53.3 \%$ & $(n=8)$ & $(n=19)$ & $61.5 \%$ & $(n=16)$ & $22.7 \%$ & $(n=10)$ \\
\hline Adequate & $40 \%$ & $(n=6)$ & $17.6 \%$ & $26.9 \%$ & $(n=7)$ & $31.8 \%$ & $(n=14)$ \\
\hline
\end{tabular}

chi-square, $p=0.018$ in males; $p=0.002$ in females 


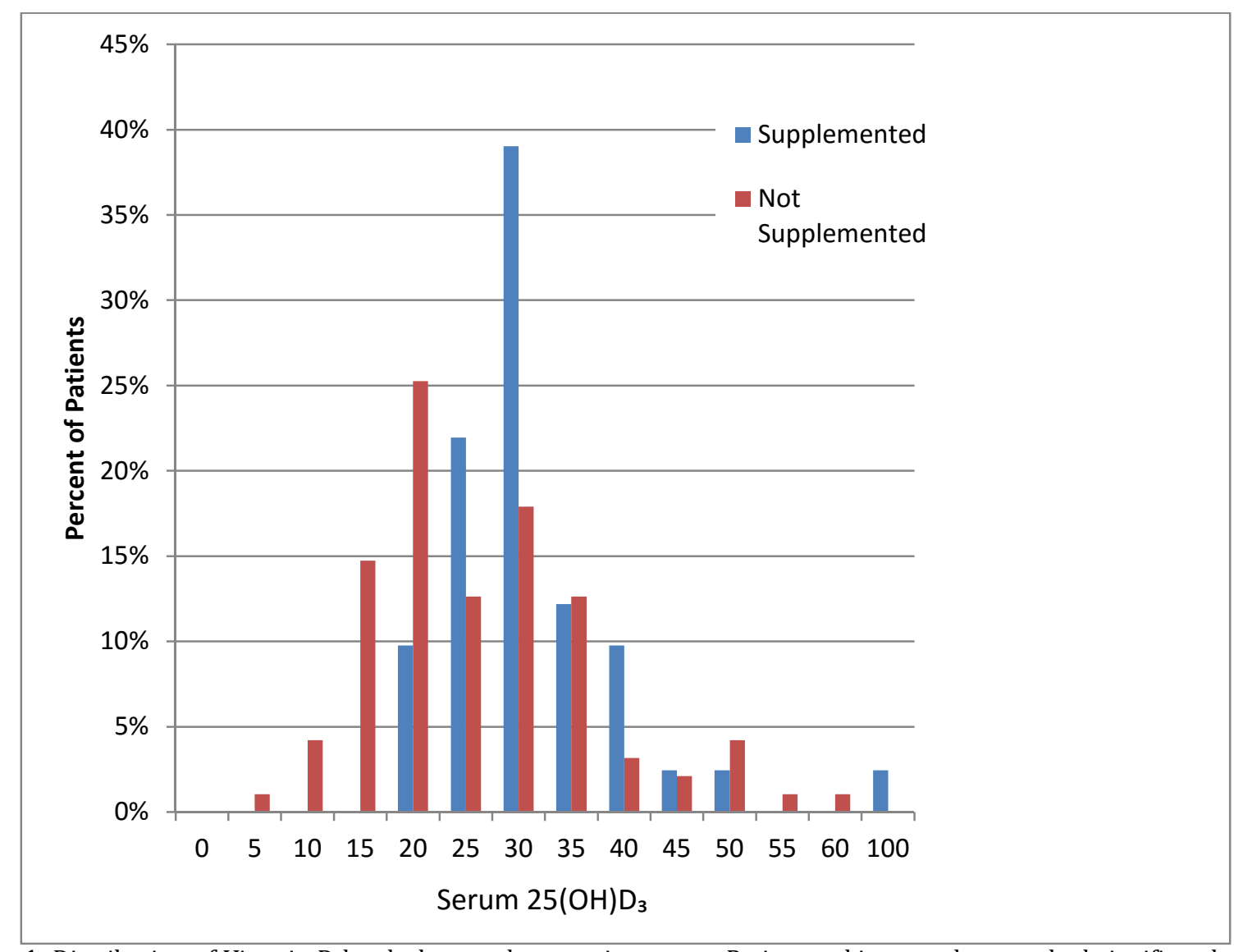

Figure 1: Distribution of Vitamin D levels, by supplementation status. Patients taking supplements had significantly higher levels of vitamin D than patients not taking supplements (Mann - Whitney $U, p=0.0016$ )

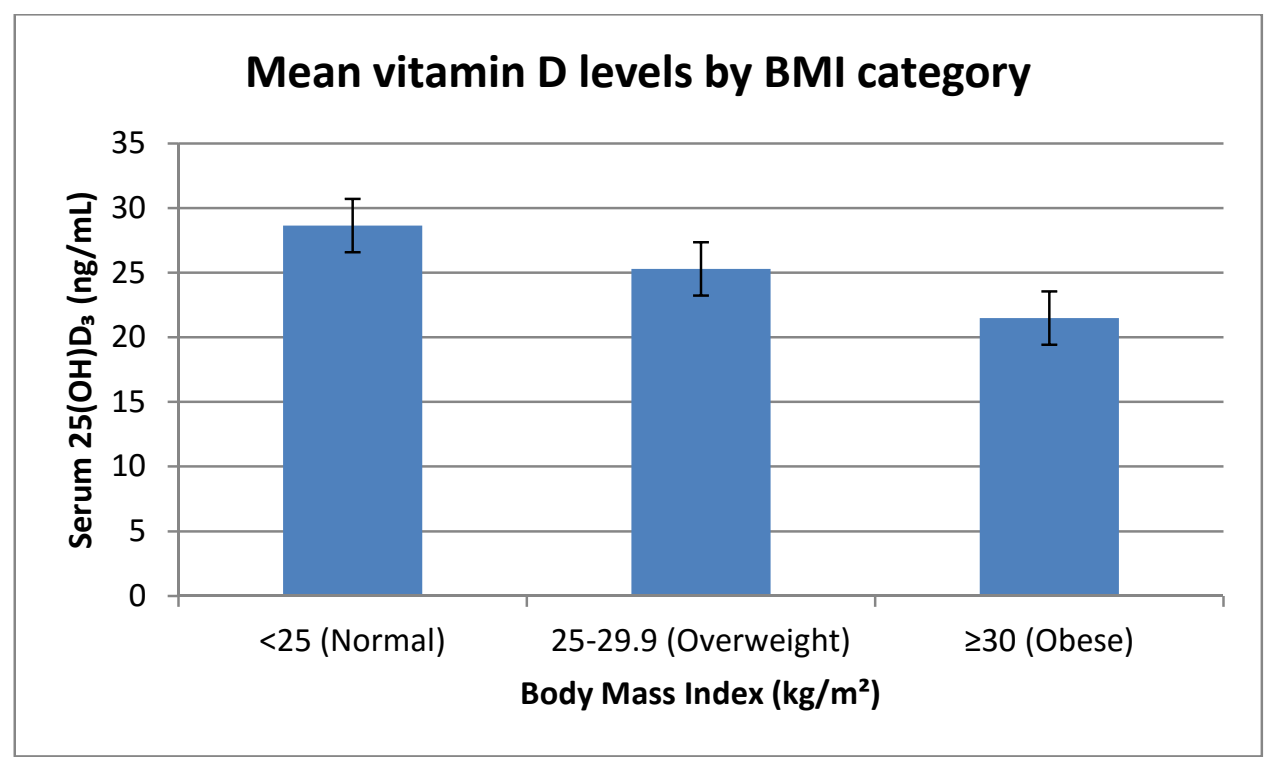

Figure 2: Mean Vitamin D levels by BMI category. Inverse relationship with lowest Vitamin D levels observed among those with highest BMIs ( F - test, $\mathrm{p}=0.003)$. 
As seen in Table 2, among males taking supplements, only $6.7 \%$ had deficient Vitamin D levels compared to $45.1 \%$ of males not taking supplementation. Furthermore, $40 \%$ of supplemented males had adequate Vitamin D levels compared to only $17.6 \%$ of non-supplemented males (chi - square, $p=0.018$ ). Among females taking supplements, only $11.5 \%$ had deficient Vitamin D levels compared to $45.5 \%$ of non supplemented females. While the proportion of females with sufficient Vitamin D levels was similar in those taking versus not taking supplements, $26.9 \%$ and $31.8 \%$, respectively, a significant difference in categorical vitamin D levels was observed in females that were supplemented compared to those not supplemented $(\mathrm{p}=$ 0.002).

Patients with a normal BMI had a mean Vitamin D level of $28.7 \mathrm{ng} / \mathrm{ml}$ compared to mean Vitamin D levels of $25.0 \mathrm{ng} / \mathrm{ml}$ for overweight and $21.5 \mathrm{ng} / \mathrm{ml}$ for obese BMI categories $(F(2,133)=5.7, p=0.004)$; with higher BMI associated with lower mean Vitamin D levels as seen in Figure 2. An analysis of variance showed significant seasonal mean vitamin $D$ variation $(F(3,132)=4.04, p=$ 0.009). Post Hoc testing found that mean Vitamin D levels were significantly lower in the winter $(M=21.9$, $\mathrm{SD}=8.8)$ and Spring months $(\mathrm{M}=22.4$, SD 10.5) than during Summer $(M=29.1, S D=10.8)$. Further, while $53.6 \%$ of the sample was found to be Vitamin D deficient during winter months, this percentage dropped to $23.3 \%$ during summer.

Predictors of insufficient or deficient vitamin D levels based on multivariate logistic regression modeling included age category (30 + years old, adjusted odds ratio $[\mathrm{OR}]=0.39,95 \%$ confidence interval $0.18-0.84$ ) and overweight/obese (BMI $25+$, adjusted OR $=2.45$, $95 \%$ confidence interval 1.08 - 5.52).

\section{Discussion}

Examination and characterization of critical health related information in the childhood cancer survivor population is fundamental to the provision of comprehensive follow - up care. It is important to note, therefore, that the findings of the current investigation indicate that the median levels of Vitamin D $(25.2 \mathrm{ng} / \mathrm{ml}$ in our sample of adult childhood cancers survivor is just above the deficiency threshold of $20 \mathrm{ng} / \mathrm{ml}$ ) and well below suggested adequate levels of $>30 \mathrm{ng} / \mathrm{ml}$. When applying thresholds for Vitamin D categories and matching age groups, patterns noted in the current study were comparable to those observed in the general population as reported in the NHANES data. ${ }^{6}$ Specifically, NHANES revealed that $32.6 \%$ of males and $37.3 \%$ of females ages $19-70$ had Vitamin D levels $<20$ $\mathrm{ng} / \mathrm{ml}$. Our study showed similar outcomes; with $36 \%$ of males and $32 \%$ of females in a matched age group reporting levels $<20 \mathrm{ng} / \mathrm{ml}$. Comparing results for only white patients ages 18 - 59 years reveals greater levels of Vitamin D deficiency in our survivor population (32.8\%) compared to NHANES (21.8\%). ${ }^{20}$ Childhood cancer survivors remain an important population requiring careful follow - up and intervention due to their high risk of chronic health conditions as a result of the late effects from their prior cancer therapies. Furthermore, emerging evidence suggests that Vitamin D levels should be greater than $30 \mathrm{ng} / \mathrm{ml}$ to achieve the desired health effects. ${ }^{5,21}$

Among survivors who reported that they were taking vitamin D containing supplements, a majority (53.3\% of males and $61.5 \%$ of females) remained insufficient and $6.7 \%$ of males and $11.5 \%$ of females continued to have deficient vitamin D levels. It is not clear whether this reflects inadequate levels of supplementation or poor adherence or a combination of these factors. To date, only a limited number of studies have examined Vitamin D deficiency and insufficiency among childhood cancer survivors. In a study by Esbenshade et $a l^{22}$, for example, Vitamin D abnormalities were studied in patients aged less than 23 years in Nashville, Tennessee who had received $>28$ days of corticosteroid therapy. Vitamin D deficiency and insufficiency rates in the Esbenshade study were found to be $15.8 \%$ and $34.5 \%$, respectively. Significant associations were also found between deficiency and BMI $>85^{\text {th }}$ percentile, older age, non Caucasian or Hispanic, and winter versus summer season. ${ }^{22}$ A study by Rosen et al. found levels of Vitamin D deficiency (14.4\%) and insufficiency (39.3\%) among patients with a mean age of 12.5 years living in Phoenix, Arizona. ${ }^{23}$; proportions similar to those found by Esbenshade et al. ${ }^{22}$ As expected those studies done in areas with warmer, sunnier climates did find a lower percentage of vitamin D deficiency.

A limited number of studies have examined vitamin D levels among adult survivors of childhood cancer. Choudhary et al. noted 29\% insufficiency $(<20 \mathrm{ng} / \mathrm{ml}$ ) among a population of pediatric cancer survivors, was comparable to that observed in the general population. ${ }^{24}$ Neville et al. found that when adjusted for gender, adult survivors of childhood cancer ages 18 - 39 had a higher proportion of Vitamin D deficiency (42.4\%) when compared to community - based controls (20.8\%). ${ }^{25}$

Multiple studies have shown decreases in fracture incidence, decreases in cancer incidence, improved glucose homeostasis, lower risk of hypertension, and a decrease in nursing home admissions in subjects with vitamin D levels greater than $30 \mathrm{ng} / \mathrm{ml}^{5,}{ }^{\text {26-28 }}$ However, the Food and Nutrition Board has recommended avoiding vitamin D levels above 50 - $60 \mathrm{ng} / \mathrm{ml}$ due to several studies showing adverse health effects. In a nested case control study, researchers found a 3 - fold greater risk of pancreatic cancer with vitamin D levels that were greater than $26.2 \mathrm{ng} / \mathrm{ml} .^{29}$ Durup et al. ${ }^{29-31}$ found a reversed J - shaped relationship between vitamin D level and all - cause mortality and for 
mortality due to cardiovascular disease, stroke, or myocardial infarction with the lowest mortality rates at vitamin D levels of approximately $30 \mathrm{ng} / \mathrm{ml}^{.29-31}$ While the US Preventative Services Task Force (USPSTF) has noted that testing of Vitamin D levels has increased by at least $50 \%$ from 2009 to 2010 , taken together, results to date suggest the need for further research to more conclusively determine optimal vitamin D levels. ${ }^{32}$

Adult survivors of childhood cancers are at an increased risk of developing chronic health conditions. ${ }^{33-35}$ Oeffinger, et al. $^{36}$ reported that $62.3 \%$ of childhood cancer survivors had at least one chronic condition with $27.5 \%$ having a severe or life - threatening condition (grade 3 or 4); most commonly seen were secondary malignant cancers, cardiovascular disease, renal dysfunction, severe musculoskeletal problems, and endocrinopathies. $^{36}$ In addition, childhood cancer survivors are known to have early mortality from secondary malignant neoplasms and cardiopulmonary events when compared to age and gender matched general population controls. ${ }^{37-40}$ We are unaware of longitudinal studies evaluating the effect of Vitamin D levels on long term complications among childhood cancer survivors. However, Vitamin D may play an important role in prevention and health maintenance for childhood cancer survivors. Future research might prospectively assess clinical outcomes and Quality of Life (QOL) among cancer survivors by category of Vitamin D levels as well as intervention studies to determine if supplementation with Vitamin D for childhood cancer survivors with insufficient/deficient levels decreases morbidity and mortality.

The current Institute of Medicine recommendations for vitamin D daily intake is 200 IU daily from birth to age 50 years, 400 IU daily for those aged 51 - 70 years, and 600 IU daily for those aged $>70$ years. However, given the controversy regarding optimal vitamin D levels, as well as individual and geographic variations, the supplement dose needed to achieve sufficient levels is also difficult to ascertain, and long - term studies need to be performed to assess the safety and efficacy of long term Vitamin D supplementation.

Limitations of the study include self-reported Vitamin D supplement use and dosage among participants. All patients, however, are questioned about their use of prescribed medications and supplements as part of the standardized clinical evaluation. For future research, improved documentation of the dose and frequency of Vitamin D supplements will be important to do a more granular analysis of the impact of Vitamin D levels in childhood cancer survivor populations. While we potentially included all patients seen for surveillance, not all patients had vitamin D levels assessed resulting in a modest sample size.
Despite the noted limitations, the present study is important in providing further evidence regarding the high prevalence of vitamin D deficiency/insufficiency among adult survivors of childhood cancers, and includes a substantial subset of persons $>40$ years of age who are generally underrepresented in childhood cancer survivor cohorts.

\section{Conclusion}

We noted a strikingly low median level of serum vitamin D among this cohort of adult survivors of childhood cancers and high proportions of survivors with insufficient and deficient vitamin D levels. Given the documented late effects associated with therapies for childhood cancer, the issue of suboptimal Vitamin D levels among childhood cancer survivors is of substantial clinical relevance.

\section{Conflict of interest}

Dr. Rokitka has received research support from Hyundai Hope on Wheels and the Roswell Park Cancer Institute Alliance Foundation related to survivorship research among pediatric cancer patients. Drs. Rokitka, Mahoney and Zevon declares that they have no conflict of interest related to the content of this manuscript. Mr. Lisac and Ms. Heffler declare that they have no conflict of interest.

Ethical approval: All procedures performed in studies involving human participants were in accordance with the ethical standards of the institutional and / or national research committee and with the 1964 Helsinki declaration and its later amendments or comparable ethical standards. This research was approved by the RPCI IRB.

\section{Funding}

This study was supported by in part by Roswell Park Cancer Institute and National Cancer Institute (NCI) grants 3P30CA01605.

\section{References}

1. Altekruse SF KC, Krapcho M, Neyman N, et al. SEER Cancer Statistics Review. 1975-2007 National Cancer Institute Bethesda, MD. 2010; Available from (based on November 2009 SEER data submission, posted to the SEER web site, 2010.) http://seer.cancer.gov/csr/1975_2007/

2. Mertens AC, Liu Q, Neglia JP, et al. Cause-specific late mortality among 5-year survivors of childhood cancer: the Childhood Cancer Survivor Study. J Natl Cancer Inst. 2008;100(19):1368-79.

3. Green DM. Long-term complications of therapy for cancer in childhood and adolescence. Baltimore, Md. Johns Hopkins University Press; 1989;16:171. 
4. Green DM. Diagnosis and management of malignant solid tumors in infants and children. Boston: Nijhoff ; Hingham, MA, USA : Distributors for North America, Kluwer Academic; 1985;10:522.

5. Vieth R. Why the minimum desirable serum 25-hydroxyvitamin D level should be 75 $\mathrm{nmol} / \mathrm{L}(30 \mathrm{ng} / \mathrm{ml})$.Best Pract Res Clin Endocrinol Metab. 2011;25(4):681-91.

6. Looker AC, Johnson CL, Lacher DA, et al. Vitamin D status: United States, 2001-2006. NCHS data brief. 2011(59):1-8.

7. Forrest KY, Stuhldreher WL. Prevalence and correlates of vitamin D deficiency in US adults. Nutr Res. 2011;31(1):48-54.

8. Holick MF. Vitamin D deficiency. N Engl J Med. 2007;357(3):266-81.

9. Woolcott CG, Wilkens LR, Nomura AM, et al. Plasma 25-hydroxyvitamin D levels and the risk of colorectal cancer: the multiethnic cohort study. Cancer Epidemiol Biomarkers Prev. 2010;19(1):130-4.

10. Wactawski-Wende J, Kotchen JM, Anderson GL, et al. Calcium plus vitamin D supplementation and the risk of colorectal cancer. N Engl J Med. 2006;354(7):684-96.

11. Ma Y, Zhang P, Wang F, et al. Association between vitamin $\mathrm{D}$ and risk of colorectal cancer: a systematic review of prospective studies. J Clin Oncol. 2011;29(28):3775-82.

12. Gandini S, Boniol M, Haukka J, et al. Meta-analysis of observational studies of serum 25-hydroxyvitamin D levels and colorectal, breast and prostate cancer and colorectal adenoma.Int J Cancer. 2011;128(6):1414-24.

13. Pittas AG, Dawson-Hughes B, Li T, et al. Vitamin $\mathrm{D}$ and calcium intake in relation to type 2 diabetes in women. Diabetes care. 2006;29(3): 650-6.

14. Krause R, Buhring M, Hopfenmuller W, et al. Ultraviolet B and blood pressure. Lancet. 1998;352(9129):709-10.

15. Hypponen E, Laara E, Reunanen A, et al. Intake of vitamin $\mathrm{D}$ and risk of type 1 diabetes: a birth-cohort study. Lancet. 2001;358(9292): 1500-3.

16. Holick MF. Sunlight and vitamin D for bone health and prevention of autoimmune diseases, cancers, and cardiovascular disease. Amer J Clin Nutr. 2004;80(6 Suppl):1678S-88S.

17. Hesseling PB, Hough SF, Nel ED, et al. Bone mineral density in long-term survivors of childhood cancer.Int J Cancer. 1998;11:44-7.

18. Wasilewski-Masker K, Kaste SC, Hudson MM, et al. Bone mineral density deficits in survivors of childhood cancer: long-term follow-up guidelines and review of the literature. Pediatrics. 2008;121(3):e705-13.
19. Halton JM, Atkinson SA, Fraher L, et al. Altered mineral metabolism and bone mass in children during treatment for acute lymphoblastic leukemia. J Bone Miner Res. 1996;11(11): 1774-83.

20. National Center for Health Statistics. National Health and Nutrition Examination Survey, 2001-2006. Available from : http://www.cdc.gov/nchs/nhanes/nhanes que stionnaires.htm. [Accessed on March 7, 2016].

21. Dawson-Hughes B, Heaney RP, Holick MF, et al. Estimates of optimal vitamin D status. Osteoporos Int. 2005;16(7):713-6.

22. Esbenshade AJ, Sopfe J, Zhao Z, et al. Screening for vitamin $D$ insufficiency in pediatric cancer survivors. Pediatr Blood Cancer. 2014;61(4): 723-8.

23. Rosen GP, Beebe KL, Shaibi GQ. Vitamin D levels differ by cancer diagnosis and decline over time in survivors of childhood cancer. Pediatr Blood Cancer. 2013;60(6):949-52.

24. Choudhary A, Chou J, Heller G, et al. Prevalence of vitamin D insufficiency in survivors of childhood cancer. Pediatr Blood Cancer. 2013;60(7):1237-9.

25. Neville KA, Walker JL, Cohn RJ, et al. The prevalence of Vitamin D deficiency is higher in adult survivors of childhood cancer. Clin Endocrinol (Oxf). 2015;82(5):657-62.

26. Bischoff-Ferrari HA. Optimal serum 25-hydroxyvitamin D levels for multiple health outcomes. Adv Exp Med Biol. 2014;810:500-25.

27. Bischoff-Ferrari HA, Giovannucci E, Willett WC, et al. Estimation of optimal serum concentrations of 25-hydroxyvitamin D for multiple health outcomes. Am J Clin Nutr. 2006;84(1):18-28.

28. Hypponen E, Power C. Vitamin D status and glucose homeostasis in the 1958 British birth cohort: the role of obesity. Diabetes care. 2006;29(10):2244-6.

29. Stolzenberg-Solomon RZ, Vieth R, Azad A, et al. A prospective nested case-control study of vitamin D status and pancreatic cancer risk in male smokers. Cancer research. 2006;66(20): 10213-9.

30. Durup D, Jorgensen HL, Christensen J, et al. A reverse J-shaped association of all-cause mortality with serum 25-hydroxyvitamin D in general practice: the CopD study. J Clin Endocrinol Metab. 2012;97(8):2644-52.

31. Durup D, Jorgensen HL, Christensen J, et al. A reverse J-shaped association between serum 25-hydroxyvitamin D and cardiovascular disease mortality - the CopD study. J Clin Endocrinol Metab. 2015;100(6):2339-46..

32. Available from http://www.uspreventiveservicestaskforce.org /Page/Document/UpdateSummaryFinal/vitami 
n-d-deficiency-screening [Accessed on March 7, 2016].

33. Zhang Y, Lorenzi MF, Goddard K, et al. Late morbidity leading to hospitalization among 5-year survivors of young adult cancer: a report of the childhood, adolescent and young adult cancer survivors research program. Int J Cancer. 2014;134(5):1174-82.

34. Geenen MM, Cardous-Ubbink MC, Kremer LC, et al. Medical assessment of adverse health outcomes in long-term survivors of childhood cancer. JAMA. 2007;297(24):2705-15.

35. Hudson MM, Ness KK, Gurney JG, et al. Clinical ascertainment of health outcomes among adults treated for childhood cancer. JAMA. 2013;309(22):2371-81.

36. Oeffinger KC, Mertens AC, Sklar CA, et al. Chronic health conditions in adult survivors of childhood cancer. N Engl J Med. 2006;355(15): 1572-82.
37. Armstrong GT, Liu Q, Yasui Y, et al. Late mortality among 5-year survivors of childhood cancer: a summary from the Childhood Cancer Survivor Study. J Clin Oncol. 2009;27(14): 2328-38.

38. Armstrong GT, Pan Z, Ness KK, et al. Temporal trends in cause-specific late mortality among 5-year survivors of childhood cancer. J Clin Oncol2010;28(7):1224-31.

39. Bhatia S, Francisco L, Carter A, et al. Late mortality after allogeneic hematopoietic cell transplantation and functional status of long-term survivors: report from the Bone Marrow Transplant Survivor Study. Blood. 2007;110(10):3784-92.

40. Lawless SC, Verma P, Green DM, et al. Mortality experiences among $15+$ year survivors of childhood and adolescent cancers. Pediatr Blood Cancer. 2007;48(3):333-8. 